\title{
La Escuela de Ingeniería Industrial presenta módulo de invernadero hidropónico climatizado automáticamente, como alternativa de producción en cadenas productivas hortifrutícolas y otras similares
}

\author{
The School of Industrial Engineering Presents Module of Automatically Weatherized \\ Hydroponic Greenhouse, as an Alternative Production in Horticultural Production \\ Chains and Other Similar Ones
}

\author{
Dennis Benavente*, Juan M. Vizcarra Rodríguez*, Percy Ruelas*, \\ Asencio Huaita*, Daniel Begazo*
}

http://dx.doi.org/10.21503/CienciayDesarrollo.2006.v7.01

\section{RESUMEN}

Los módulos de invernaderos hidropónicos climatizados automáticamente para propiciar un sistema de producción alternativo a la agricultura tradicional permiten mejorar el balance tecnológico en las cadenas productivas de distintas hortalizas y vegetales similares, reduciendo las grandes brechas entre tecnologías y procesos usados actualmente en la producción de estos vegetales en la región sur del Perú y los utilizados en otros países considerados líderes en la aplicación de las mejores prácticas dentro de estas cadenas productivas a nivel mundial.

Los módulos mencionados permitirán el logro de altos índices de productividad y de bioseguridad, reducir costos de operación al mínimo y llegar a ciclos de cultivo mínimos. Además, con el control automatizado y en tiempo real de las variables de operación involucradas en el control del microclima, como son la temperatura, la humedad, el $\mathrm{pH}$, la luminosidad, los niveles de $\mathrm{CO}_{2}$, la conductividad eléctrica, etc., se garantiza una producción de vegetales altamente competitiva en cantidad, calidad y costo, lo cual, a su vez, permitirá acceder a mercados locales, nacionales e internacionales.

Actualmente, es de suma importancia contar con sistemas productivos que garanticen una producción limpia y la no contaminación del medio ambiente, siendo precisamente la contaminación una constante observada en nuestra localidad, motivada sobre todo por el uso de aguas contaminadas del río Chili, el empleo de agroquímicos prohibidos y la utilización de suelos altamente degradados y contaminados.

El módulo propuesto permite cultivar hortalizas hidropónicas con bajo contenido de nitrato durante todo el año, en zonas áridas y bajo condiciones controladas de luz, temperatura y humedad, utilizándose para tal efecto un sistema de automatización que consiste en un núcleo central con mandos de sensores y electroválvulas que se encuentran en estado de prueba, y realizando además modelamientos para su mayor eficiencia y eficacia dentro de una red industrial.

En el módulo se viene utilizando, en fase de experimentación, la lechuga tipo Carola, siendo nuestras proyecciones de rendimiento del orden de 90 lechugas $/ \mathrm{m}^{2}$ en 3 niveles, aprovechando la máxima radiación solar que recibe Arequipa. En otros invernaderos, el rendimiento es de 12 a 30 lechugas $/ \mathrm{m}^{2}$.

El módulo está implementado siguiendo el sistema de hidroponía no circulante de IMAI de la AVRDC (Instituto de Investigación de Vegetales del Asia), con la aplicación de soluciones nutritivas de la FAO (Fondo para la Alimentación de las Naciones Unidas).

Palabras clave: invernadero hidropónico, bioseguridad, radiación solar.

* Docente de la Universidad Alas Peruanas, filial Arequipa. 


\section{ABSTRACT}

To produce modules of automaticaly weatherized hydroponic greenhouses, to encourage an alternative production system to traditional agriculture, which enables us to improve the technological balance for the Production Chains of the different and similar vegetables, reducing the big gaps between technologies and processes used at present in the production on these vegetable in the southern region of Perú and those used in other countries considered to be leaders the application of the best methods in these prodcution chains around the world.

The mentioned modules will enable us to get in the best way optimized metrics of performance such as high levels of productivity and biosecurity, minimum operative costs, minimum cultivation cycles, etc., which are reached through the real-time and automatized control of the operation variables involved in the control of microclimates such as temperature, humidity, $\mathrm{pH}$, luminosity, $\mathrm{CO}_{2}$, electrical conductivity, etc., with which you can guarantee a highly competitive production of vegetables in quantity, quality and cost that will let us get into local, national and international markets.

Today, it is really important to have production systems which guarantee a clean production which does not pollute the environment, being this last condition a constant observed in our town, because of the use of polluted water from the Chili river, the use of prohibited agrochemicals, the use of highly gradient and polluted soils.

This proposed module will enable us to grow hydroponic vegetables with a low content of Nitrate; during the whole year, en arid regions and under controlled conditions of: light, temperature and humidity, using for this purpose an automatization system which consists of a central unit with commanding sensors and electro-valves which are in trial performing modelings for a better efficiency and effectiveness of performance in an industrial Net.

In this module Carola lettuce is being used in the experimentation phase, being our projections of productivity 90 lettuces $/ \mathrm{m}^{2}$ in three levels, taking advantage of the maximum solar radiation that Arequipa has, whereas in other greenhouses the productivity is $12-30$ lettuces $/ \mathrm{m}^{2}$.

This module is implemented with a non-circulaitng hydroponic system of Imai of the AVRDC (Vegetable Investigation Institute of Asia) with the applications of Nutrient Solutions of the FAO (United Nations).

Key words: hydroponic greenhouses, biosecurity, solar radiation.

\section{INTRODUCCIÓN}

Hace 2 años, la Escuela de Ingeniería Industrial emprendió este proyecto con el apoyo de sus docentes y alumnos. El objetivo era facilitar un medio de producción limpia y eficiente, minimizando el daño ambiental y aprovechando ventajas en el área de construcción de invernaderos, con la implementación de un invernadero tipo capilla de $45 \mathrm{~m}^{3}$ y que cumpla con los estándares internacionales.

Un indicador de contaminación en una ciudad es la calidad de agua de sus ríos. En Arequipa, son 8000 las hectáreas regadas directamente con aguas servidas, y por lo tanto la contaminación es evidente, sobre todo si se sabe que las concentraciones de coliformes fecales exceden el límite (1000 NMP) (GEO AREQUIPA, 2005). La solución está planteada a largo plazo, proyectándose para el 2015 una planta de tratamiento de aguas servidas. Frente a la demanda inmediata y mediata de verduras y hortalizas sanas, libres de contaminación y de bajas concentraciones en nitratos -condiciones exigidas por los sistemas de distribución y comercialización modernos-, los supermercados empiezan a dominar los mercados locales. En tal sentido, el sistema de producción propuesto es el que tendrá un éxito garantizado frente a la agricultura tradicional. 
El módulo de invernadero cuenta con 4 camas, habiéndose establecido un sistema escalonado de producción sistematizado. En este momento se está en una etapa de prueba y de afinamiento, con los porcentajes de humedad apropiados y combinados con las 11 horas de luz que recibe el invernadero, el mismo que está orientado en la dirección de la luz solar, con el propósito de maximizar el rendimiento al interior del modulo. La proyección es contar con 3 niveles de un monocultivo de hortalizas eficientes y limpias, con temperaturas de microclima que oscilarían entre $14^{\circ} \mathrm{C}$ y 22 ${ }^{\circ} \mathrm{C}$, y con sistemas de regulación que permitan, a futuro, cambiar los patrones internos.

\section{MATERIAL Y MÉTODOS}

Se preparó un módulo de invernadero tipo capilla, con medidas aprobadas por el Instituto de Vegetales del Asia: $3 \mathrm{~m}$ x $6 \mathrm{~m}$ con una altura máxima de 3,50 m, confeccionado con ángulos de fierro negro y con ventanales de vidrio. Se instalaron 3 camas para producción, de $75 \mathrm{~cm}$ por $150 \mathrm{~cm}$ y $10 \mathrm{~cm}$ de alto, con capacidad de 115 litros de solución nutritiva.

El método utilizado para la producción limpia y eficiente es el sistema de hidroponía no circulante, que consiste en la aplicación, como sustrato, de una solución nutritiva constituida por N: 250 p.p.m, P: 90 p.p.m, K: 250 p.p.m y Mg: 150 p.p.m. Utiliza un semillero en potes de 4 " de PVC, con sustrato de cascajo de 4 a $6 \mathrm{~mm}$ y con siembra de semilla de lechuga híbrida.

Respecto a la automatización, podemos mencionar los componentes de nuestro prototipo de red industrial:

- Protocolo de comunicación: Protocolo MODBUS

- Sistema operativo: Windows XP - SP2

- Software de aplicación: Factory Link
- Tipo de red

- Según su tecnología (Broadcast)

- Según su extensión ( LAN)

- Según su procesamiento (Centralizada)

- Según su conmutación (Conmutación de circuitos y conmutación de paquetes)

- Topología de la red

- Bus

- Transmisión en serie (velocidad de transmisión: 9600 baudios)

- Norma utilizada: RS485

- Transmisión asíncrona

En este proceso de automatización, hemos podido determinar, a través del análisis químico de la solución nutritiva, la concentración de cada uno de los elementos nutritivos en la solución. En este proceso se utilizaron las variables $\mathrm{pH}$ y de conductividad eléctrica, medidas por nuestros equipos de calibración y que nos permitieron manejar las condiciones necesarias para el desarrollo sano de las hortalizas y vegetales similares a ser producidos en el módulo.

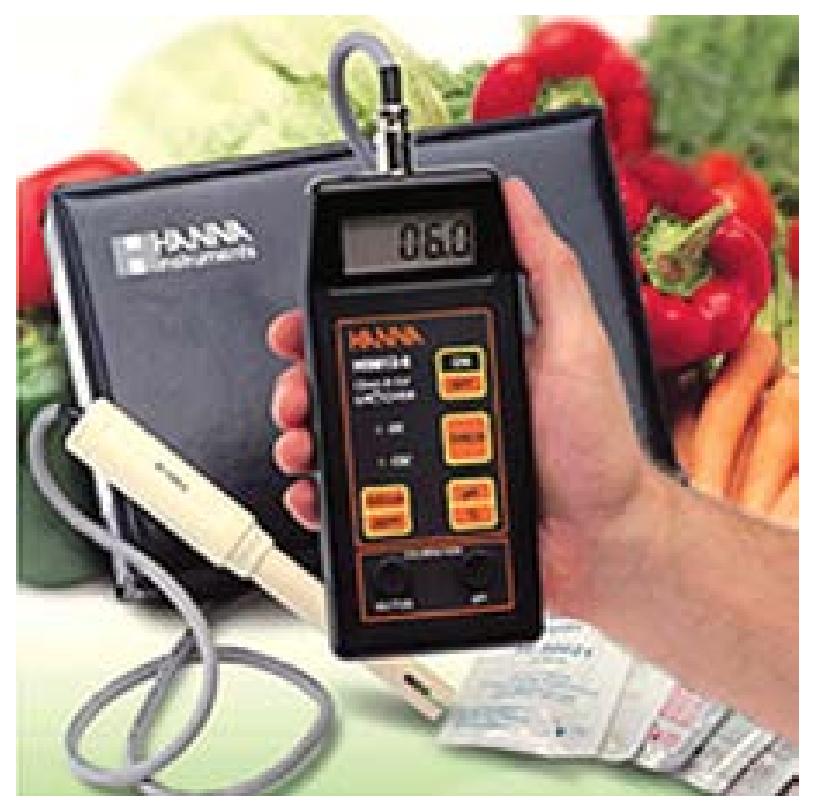

Figura 1. Equipo de calibración de variables.

Según la FAO, los siguientes son los parámetros de cultivo para el caso de lechugas. 
Tabla 1. Parámetros FAO para el cultivo de lechugas hidropónicas.

\section{ANTECEDENTES DEL CULTIVO}

\author{
Rango de temperatura óptimo de germinación \\ Tiempo aproximado de germinación \\ $\mathrm{N}^{\circ}$ aproximado de semilla/grano \\ Longevidad de la semilla \\ $\mathrm{N}^{\circ}$ plantas $/ \mathrm{m}^{2}$ en sistema "NFT" \\ Tiempo aproximado de trasplante a cosecha
}

SISTEMA "NFT"

\section{SOLUCIÓN NUTRITIVA}

Factor de conductividad

$\mathrm{pH}$

Consumo de solución aproximado por planta

\author{
$4,5-27^{\circ} \mathrm{C}$ \\ $6-12$ días \\ 800 \\ 3 años \\ 22 - 24 según el cultivar \\ 25 - 40 días
}

\section{CANAL DE CULTIVO}

Tipo de canal

Ancho mínimo de canal
$15-25$

$5,5-6,5$

0,25 litros/planta

$6 \mathrm{~cm}$

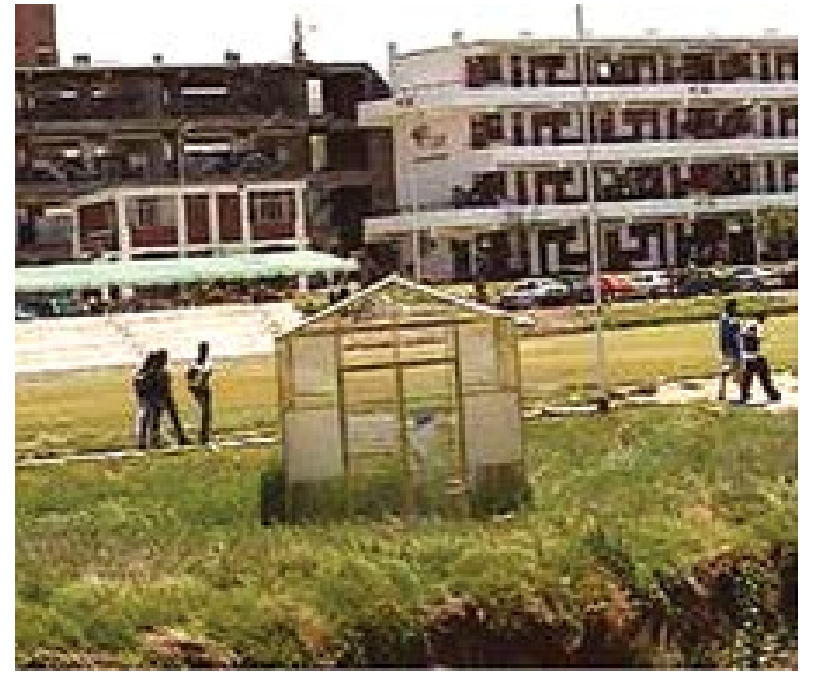

Figura 2. Módulo de invernadero hidropónico.

Se tiene la electroválvula a.c que permite el paso de agua al invernadero, una electrobomba que servirá para realimentar las reservas de agua en las camas donde se cobijan las lechugas, mi-

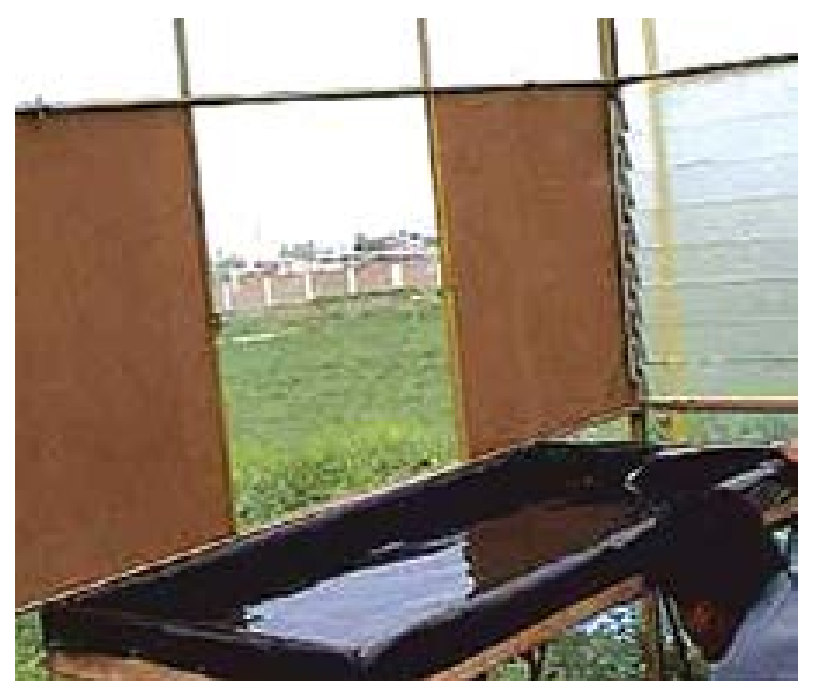

Figura 3. Instalación de camas productivas.

croaspersores para reducir el efecto de la alta temperatura en el invernadero, y los calefactores que nos permitirán mantener el ambiente templado del mismo. 
Funcionamiento automático

del invernadero

- Primer paso: Accionar la electrobomba para que ingrese el agua al tanque principal. Luego, cuando el agua alcanza el nivel deseado (detectado por el sensor de nivel), se procede a desactivar la electrobomba.

- Segundo paso: Si el valor de la temperatura es mayor a $30^{\circ} \mathrm{C}$, se acciona la electroválvula de microaspersión, y en caso de que sea menor a $4^{\circ} \mathrm{C}$, se accionan los calefactores.

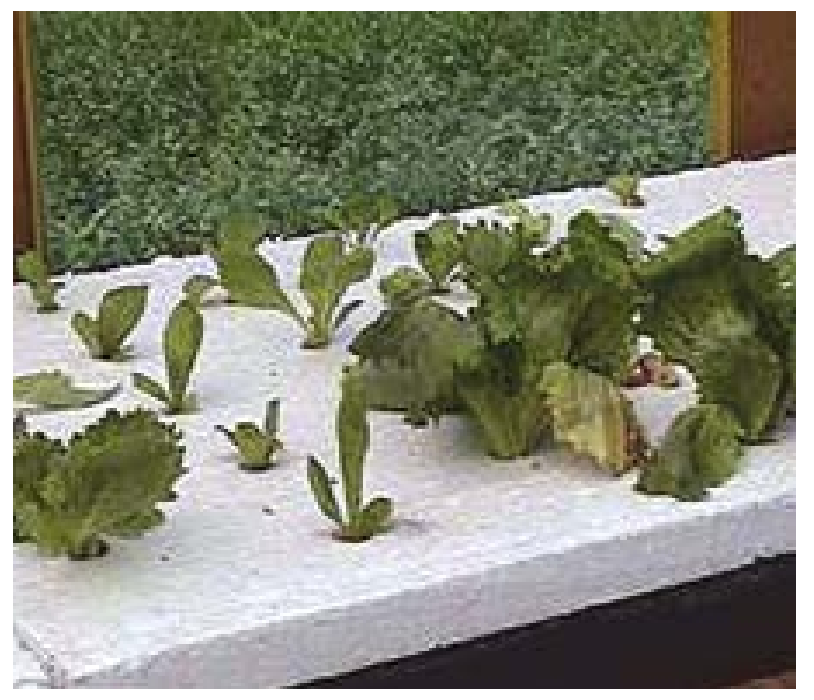

Figura 4. Cama en producción.

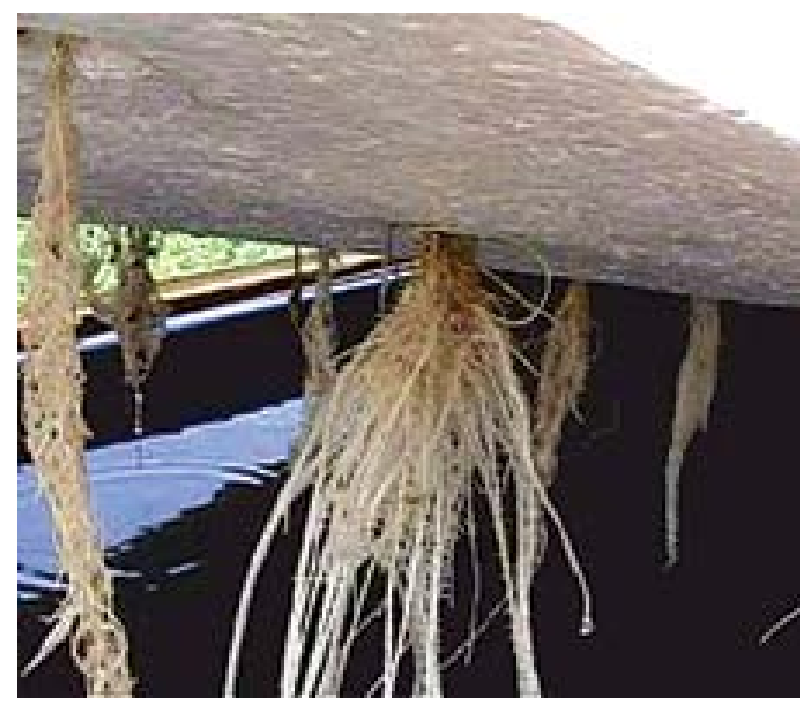

Figura 5. Sistema radicular.

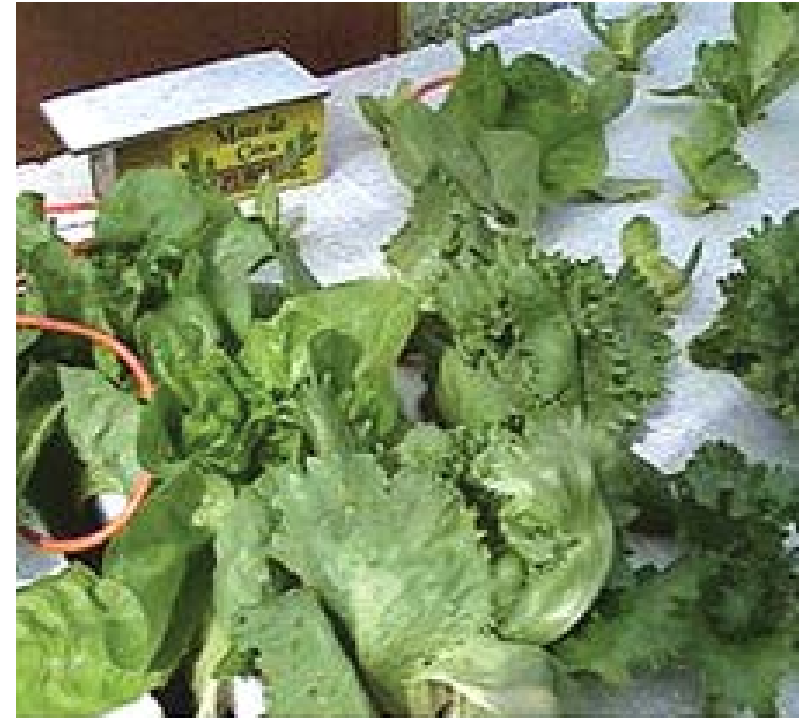

Figura 6. Desarrollo de la hortaliza al final del ciclo.

- Tercer paso: Si el valor de la humedad relativa es menor a $7 \%$, se acciona la electroválvula de microaspersión, y si es mayor a $80 \%$, se encienden los calefactores.

Se tendrá siempre en mediación un sensor de luminosidad para efectos de uso de luz artificial durante la noche, buscando acelerar el proceso y así acortar el ciclo de cultivo.

\section{RESULTADOS}

En la fase experimental de este módulo se han obtenido resultados preliminares satisfactorios en las siguientes condiciones de microclima: temperatura promedio de $18{ }^{\circ} \mathrm{C}$, humedad relativa de $60 \%$ y 11 horas de luz diarias. Se han obtenido rendimientos de 25 a 30 unidades por $\mathrm{m}^{2}$ en un nivel, proyectando la obtención de 90 lechugas $/ \mathrm{m}^{2}$ en tres niveles. Los valores de las concentraciones de nitratos han sido bajos, valores que han sido verificados con el análisis de di-fenilamina en lechugas en cosecha, las mismas que presentan un característico color verde claro. 


\section{DISCUSIÓN}

Bruce y Nichols (2000) establecieron que en los supermercados más importantes del mundo se produce una gran demanda de lechugas con bajas concentraciones de nitritos, utilizadas sobre todo en la preparación de ensaladas. Nuestra realidad nos dice que el $84 \%$ de hortalizas del mercado muestra un resultado positivo a la contaminación por heces fecales y otros elementos (MPA, 2003). Ante esta alarmante situación, el cultivo hidropónico de lechugas en los módulos propuestos se convierte en una excelente alternativa, toda vez que permitirá obtener un producto saludable, bajo en nitritos y rico en vitamina $\mathrm{A}$.

\section{BIBLIOGRAFÍA}

1. Instituto de Vegetales del Asia (AVRDC). 1990.

2. Bruce y Nichols. Agricultura de las Américas. Junio de 2000.

3. GEO Arequipa. Perspectivas del medio ambiente urbano. 2005.

4. MPA. Análisis de la Municipalidad Provincial de Arequipa. 2003. 